\title{
A Study on the Diversity and Relative Abundance of Insect Fauna in Wukari, Taraba State, Nigeria
}

\section{Okrikata Emmanuel*, Yusuf Olajumoke Anuoluwa}

\author{
Department of Biological Sciences, Federal University Wukari, Taraba State, Nigeria \\ *Corresponding Author E-mail: eokrikata@gmail.com
}

Received: 20 January 2019, Revised 19 February 2019, Accepted: 23 February 2019

\begin{abstract}
A field survey was conducted in Wukari, Taraba State to assess the diversity and abundance of insect species in selected habitats (residential, open field made up of grassland and an agro ecosystem). Sampling were done biweekly using light, pitfall and yellow pan traps set in 3 replicates, $30 \mathrm{~m}$ apart. Insects recovered were wet preserved in $70 \%$ ethanol except butterflies and moths. Representative samples were taken to the Insect Museum of Ahmadu Bello University Zaria for identification. A total of 4,501 insects spread across 9 orders, 34 families and 77 species were recovered. The most dominant order was Coleoptera with a relative abundance of (44.41\%) and, the least was Orthoptera (0.84\%). The most dominant insect species are Heteronychus mossambicus (11.44\%) followed by Termes sp. (7.77\%) and, Goryphus sp. (7.71\%). Chlaenius decipiens, Cheilomenes sulphurea, Copris sp., Cicindela sp., Pseudantheraea sp., Derobranchus geminatus, Glaurocara townsendi, Camponotus perrisi, and Gryllus bimaculatus were the rare species with relative abundance of $0.02 \%$. Species richness is based on number of individual insects measured. The highest species diversity was observed in the order Coleoptera (Shannon $\mathrm{H}^{\prime}=2.547$ ) while, Isoptera was the least $\left(\mathrm{H}^{\prime}\right.$ $=0.00)$. However, the highest species evenness was observed in the order Isoptera $\left(\mathrm{E}^{\prime}=\right.$ 1.00). Fisher-alpha $(\alpha)$ index of diversity shows that the agro ecosystem has the highest index of diversity ( $\alpha=14.24$ ) while, the residential area had the least ( $\alpha=11.9$ ). This study therefore, brings to the fore the diversity and abundance of insects in Wukari and underscores the need for a more intensive study and for sustainable actions to be taken in conserving beneficial rare species while, managing the abundant pestiferous ones.
\end{abstract}

Key words: Abundance, Diversity, Fisher-alpha $(\alpha)$, Insects, Jaccard's similarity index, Margalef (d), Shannon index (H').

\section{Introduction}

Insects are important because of their diversity, ecological role, and influence on agriculture, human health, and natural resources (Berenhaum, 1995; Adetundan et al., 2005; Premalatha et al., 2011). They have been used in landmark studies in biomechanics, climate change, developmental biology, ecology, evolution, genetics, paleolimnology, and physiology. They make up more than $58 \%$ of the known global biodiversity. They inhabit all habitat types and play major roles in the function and stability of terrestrial and aquatic ecosystems (Godfray, 2002). 
Insects are closely associated with our lives and affect the welfare of humanity in diverse ways. At the same time, large numbers of insect species, including those not known to science, continue to become extinct or extirpated from local habitats worldwide (Miller et al., 2001). The diversity of insect species is a function of the environmental condition (Yi et al., 2012).

Wukari is a richly agrarian community in the North eastern part of Nigeria. The diversity and abundance of insects in Wukari has hardly been studied. Insect biodiversity studies conducted in Nigeria have largely been on the insects' diversity of specific orders and/or species of insects. Few have considered the insect community altogether (Meddler, 1980). Aside inadequate taxonomic knowledge, the ecological knowledge of insects in Nigeria is not well understood as distribution and abundance of many insect species in the country are unknown and their ecosystem services mostly assumed (Kato et al., 2000). Anthropogenic activities have contributed to the movement and spread of invasive insects into the different habitats with many of them having Agricultural, Medical and Veterinary implications (Wardle et al., 2002).

The current study is designed for the very first time to document diversity and abundance of insects in Wukari, Taraba State, Nigeria. This information is not only useful for Agricultural, Medical and Veterinary purposes, but will also probably for the very first time, give an insight into the insect species richness of wukari; an information that is very critical for management and conservation purposes (Mazon et al., 2008).

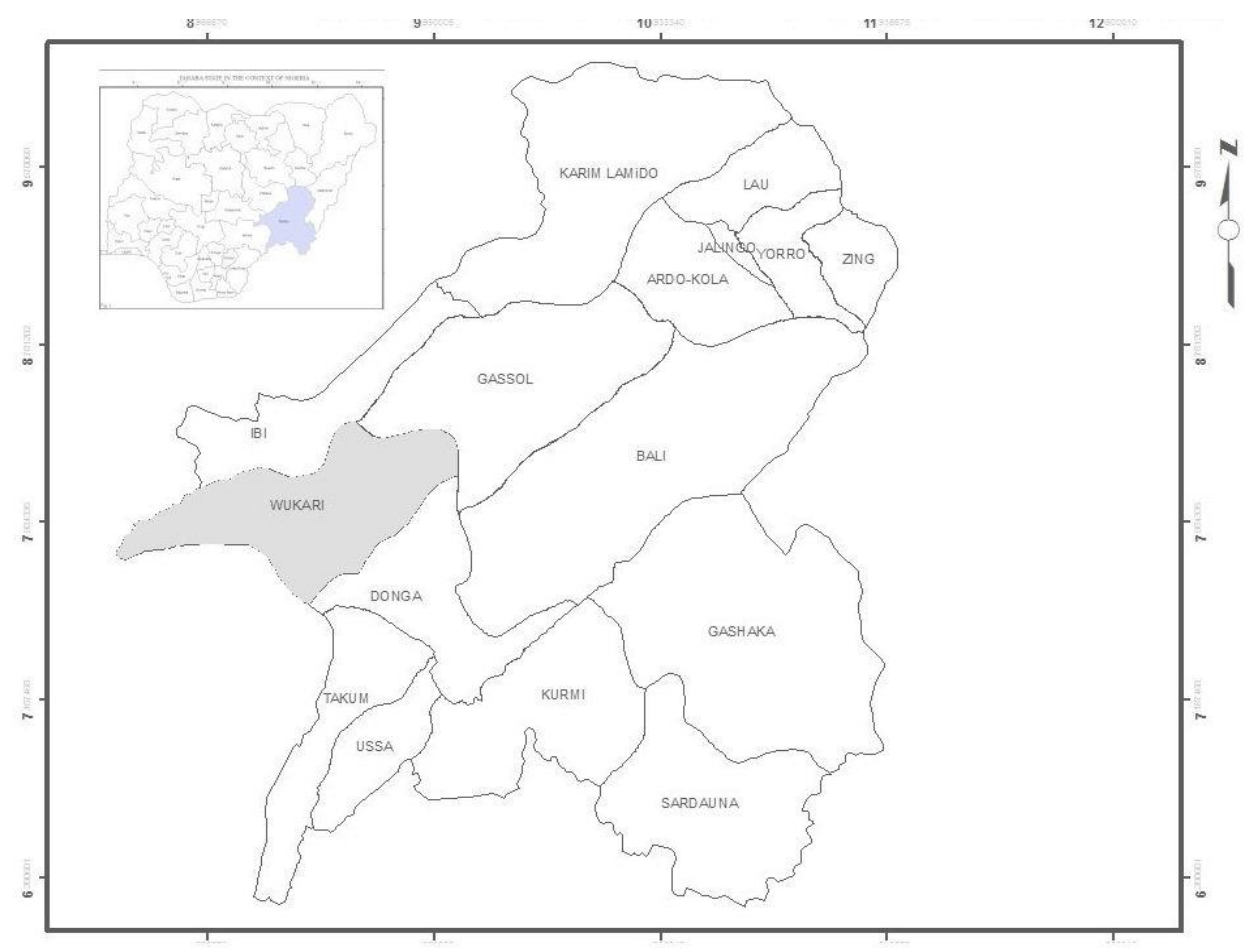

Source: Satellite Maps (2015)

Figure 1. Map of Taraba State in Nigeria, showing Wukari

The habitats/locations that were sampled are;

1. Agro-ecosystem (A farm land of about ten (10) hectares used for all year round farming)

2. Open field (A Grassland community behind Federal University Wukari, football field) 
3. Residential area (Hostel and Staff Quarters environment of Federal University Wukari).

\section{Insect sampling/collection technique}

The field survey was conducted from March to May, 2016. Insect's sampling was done biweekly using the following insects sampling techniques (Campos et al, 2000);

1. Pitfall trap: This was used to collect ground dwelling insects (Axmacher et al., 2004). A double cup design of pitfall trap with a length of $11 \mathrm{~cm}$ and, $10 \mathrm{~cm}$ wide was used in which a hole is dug and two (2) containers are placed in a dug hole and soil is packed around it to the level of the rim of the inner container (Sabu et al., 2010).

The inner cup was a removable container that allow for setting and servicing of the trap. The outer cup keeps the hole from back filling with soil. An elevated wooden tripod stand $(5 \mathrm{~cm}$ above the ground level) was placed over the pitfall to keep off water, falling debris and small rodents. Water and 2\% mild detergent were used as a killing agent (Winder et al., 2001). The content of the trap was serviced after 48 hours. By pouring the content through a sieve and rinsed with gently running water and preserved in a container containing $70 \%$ ethanol.

2. Yellow pan trap: A yellow plastic dish of $6 \mathrm{~cm}$ length and $12 \mathrm{~cm}$ wide containing a mixture of water with $2 \%$ mild detergent which breaks the surface tension of the water was placed $25 \mathrm{~cm}$ above the ground level. Flying insects landing on the surface of the water were trapped (Roulston et al., 2007; Saunder et al., 2013). The trap was set up for a period of twelve (12) hours ( 6 am to 6 $\mathrm{pm})$. Insects collected were poured into a sieve and rinsed with gently running water and then preserved in a container containing $70 \%$ ethanol.

3. Light trap: This was set by sinking two (2) nails into a tree, $10 \mathrm{~cm}$ apart with the bottom one at $3 \mathrm{~m}$ above ground level. The light source was tied on the first nail up, while the container of $17 \mathrm{~cm}$ length and $16.5 \mathrm{~cm}$ wide containing the mixture of water with $2 \%$ mild detergent was tied to the second nail just below the light source. Insects that fly onto the light source fall into the container and are trapped (Kato et al., 2000). The trap was set in the evening (6 pm) and serviced in the morning $(6 \mathrm{am})$. The insects collected were poured into a sieve and rinsed with gently running water and, preserved in 70\% ethanol. All traps were set biweekly in three (3) replicates in each habitat and were spaced about 15 meters from each other (Frank, 2006).

\section{Preservation of insects collected}

All insects collected were preserved by immersion in 70\% ethanol. However, insects like moths that have scales on their wings were preserved dry in a tight container containing Silica gel. Representative samples were preserved in the Biology Laboratory, Federal University Wukari, for future reference.

\section{Identification of insect samples}

All insects collected were taken for identification at the insect museum centre of Ahmadu Bello University Zaria, Kaduna State, Nigeria.

\section{Data analysis}

The following Biodiversity indices were computed using Past3 software;

a. Abundance of insect species.

b. Relative Abundance of insect species,

c. The Shannon Diversity Index ( $\left.\mathrm{H}^{\prime}\right)$ was used to compute the ecosystem diversity index.

d. Jaccard's Similarity Index. 
Shannon index $\left(\mathrm{H}^{\prime}\right)$ was used in calculating $\mathrm{t}^{\prime}$ to test for significant difference in diversity of insect species between the habitats surveyed.

\section{Results}

\section{Diversity and abundance of insects in the study area}

Table 1 show the diversity and abundance of insect species recovered in the selected habitats. A total of 4,501 insects belonging to 77 species, 34 families and 9 orders were recorded. The largest number of insect species (69) was recovered from farm land, and the least (56) were recovered from the open field. Across the habitats, Heteronychus mossambicus, had the highest abundance (515) followed by Termes sp. (350) and Goryphus sp. (347), the least abundant (rare) insect species include Chaenius decipiens, Cheilomenes sulphurea and, Derobrachus geminatus.

Table 1. Diversity and abundance of insect species in the study area

\begin{tabular}{|c|c|c|c|c|c|c|}
\hline Order & Famiy & Genus/species & RA & OF & $\mathbf{A G}$ & Total \\
\hline \multirow[t]{26}{*}{ Coleoptera } & Brentidae & Cylas brunneus Fab. & & & & \\
\hline & & & 0 & 11 & 24 & 35 \\
\hline & Carabidae & Arsinoe biguttata & & & & \\
\hline & & $\begin{array}{c}\text { Chaud. } \\
\text { Aulacoryssus }\end{array}$ & 16 & 37 & 49 & 102 \\
\hline & & aciculatus Dej. & 1 & 8 & 22 & 31 \\
\hline & & Aulacoryssus sp. & 12 & 18 & 27 & 57 \\
\hline & & Callida decora Fab. & 0 & 15 & 11 & 26 \\
\hline & & Callida sp. & 0 & 0 & 2 & 2 \\
\hline & & Chlaenius decipiens & & & & \\
\hline & & Dufour. & 1 & 0 & 0 & 1 \\
\hline & & $\begin{array}{c}\text { Cicindela sp. } \\
\text { Dichaetochilus vagans }\end{array}$ & 0 & 1 & 0 & 1 \\
\hline & & Dej. & 22 & 12 & 26 & 60 \\
\hline & & Edagroma sp. & 16 & 15 & 0 & 31 \\
\hline & & Paussus sp. & 2 & 1 & 5 & 8 \\
\hline & Cerambycidae & Derobrachus & & & & \\
\hline & & $\begin{array}{c}\text { geminatus LeConte } \\
\text { Paroeme nigripes }\end{array}$ & 0 & 0 & 1 & 1 \\
\hline & & Auriv. & 58 & 69 & 57 & 184 \\
\hline & Chrysomelidae & $\begin{array}{c}\text { Stobiderus sp. } \\
\text { Aspidomorpha } \\
\text { nigromaculata Herbt. }\end{array}$ & 10 & 13 & 47 & 70 \\
\hline & & & 2 & 1 & 2 & 5 \\
\hline & Coccinellidae & Cheilomenes & & & & \\
\hline & Curculionidae & $\begin{array}{l}\text { sulphurea Oliv. } \\
\text { Alcidodes brevirostris }\end{array}$ & 0 & 0 & 1 & 1 \\
\hline & & Boh. & 7 & 3 & 3 & 13 \\
\hline & Curculionidae & Colliuris sp. & 7 & 24 & 26 & 57 \\
\hline & Elateridae & Melanoxanthus sp. & 40 & 0 & 1 & 41 \\
\hline & & Prosephus sp. & 43 & 51 & 32 & 126 \\
\hline & Hesteridae & Hister sp. & 21 & 0 & 3 & 24 \\
\hline
\end{tabular}




\section{Contine: Table 1}

\begin{tabular}{|c|c|c|c|c|c|c|}
\hline Order & Family & Genus/species & $\mathbf{R A}$ & OF & AG & Total \\
\hline \multirow[t]{9}{*}{ Coleoptera } & \multirow[t]{6}{*}{ Scarabaeidae } & Anomala mixta Fab. & 38 & 70 & 42 & 150 \\
\hline & & $\begin{array}{c}\text { Copris sp. } \\
\text { Heteronychus } \\
\text { mossambicus }\end{array}$ & 0 & 0 & 1 & 1 \\
\hline & & Peringuey. & 137 & 106 & 272 & 515 \\
\hline & & Onthophagus sp. & 35 & 79 & 85 & 199 \\
\hline & & Serica sp. & 7 & 59 & 47 & 113 \\
\hline & & $\begin{array}{c}\text { Schizonycha africana } \\
\text { Castel. }\end{array}$ & 51 & 9 & 31 & 91 \\
\hline & \multirow[t]{3}{*}{ Tenebrionidae } & Derophaerus sp. & 0 & 11 & 2 & 13 \\
\hline & & Disonycha sp. & 15 & 28 & 55 & 98 \\
\hline & & Tenebriodes sp. & 37 & 0 & 34 & 71 \\
\hline \multirow[t]{3}{*}{ Dictyoptera } & \multirow[t]{3}{*}{ Blattidae } & Blattella sp. & 2 & 8 & 7 & 17 \\
\hline & & Deropeltis sp. & 9 & 8 & 3 & 20 \\
\hline & & Gyna costalis Walk. & 8 & 22 & 3 & 33 \\
\hline \multirow[t]{10}{*}{ Diptera } & $\begin{array}{c}\text { Asilidae } \\
\text { Calliphoridae }\end{array}$ & $\begin{array}{l}\text { Ommatius sp. } \\
\text { Chrysomyia albiceps }\end{array}$ & 0 & 5 & 2 & 7 \\
\hline & \multirow{5}{*}{ Muscidae } & $\begin{array}{l}\text { Wield. } \\
\text {. }\end{array}$ & 0 & 1 & 6 & 7 \\
\hline & & $\begin{array}{c}\text { Morellia nilotica } \\
\text { Loew } \\
\text { Musca domestica Lin. }\end{array}$ & 0 & 0 & 2 & 2 \\
\hline & & Musca sorbens Wied. & 25 & 25 & 27 & 77 \\
\hline & & & 3 & 2 & 1 & 6 \\
\hline & & Musca sp. & 0 & 0 & 2 & 2 \\
\hline & \multirow[t]{2}{*}{$\begin{array}{l}\text { Scarcophagidae } \\
\text { Stratiomiidae }\end{array}$} & $\begin{array}{l}\text { Scarcophaga sp. } \\
\text { Acrodesmia }\end{array}$ & 0 & 3 & 0 & 3 \\
\hline & & $\begin{array}{l}\text { pennicornis Berri. } \\
\text { Glaurocara townsendi }\end{array}$ & 0 & 0 & 2 & 2 \\
\hline & \multirow{2}{*}{ Tachinidae } & $\begin{array}{c}\text { Emden. } \\
\text { Latiginella rufogrisea } \\
\text { Villeneuve }\end{array}$ & 0 & 0 & 1 & 1 \\
\hline & & & 18 & 7 & 55 & 80 \\
\hline
\end{tabular}

\section{Contine: Table 1}

\begin{tabular}{|c|c|c|c|c|c|c|}
\hline Order & Family & Genus/species & $\mathbf{R A}$ & $\mathbf{O F}$ & $\mathbf{A G}$ & Total \\
\hline \multirow[t]{6}{*}{ Hemiptera } & Flatidae & $\begin{array}{c}\text { Cryptoflata } \\
\text { unipunctuntata Oliv. }\end{array}$ & & & & \\
\hline & & & 0 & 0 & 6 & 6 \\
\hline & Pentatomidae & $\begin{array}{c}\text { Aspavia acuminata } \\
\text { Mont. }\end{array}$ & 1 & 0 & 2 & 3 \\
\hline & Reduviidae & $\begin{array}{c}\text { Piezodorus sp. } \\
\text { Coranus lugubris Stal. }\end{array}$ & 2 & 26 & 27 & 55 \\
\hline & & & 1 & 0 & 4 & 5 \\
\hline & & Oncocephalus sp. & 1 & 3 & 3 & 7 \\
\hline \multirow{6}{*}{ Hymenoptera } & Apidae & Halictus sp. & 5 & 16 & 9 & 30 \\
\hline & Braconidae & Apanteles sp. & 6 & 7 & 2 & 15 \\
\hline & & Braunsia sp. & 2 & 0 & 2 & 4 \\
\hline & & Ipiaulax sp. & 35 & 38 & 40 & 113 \\
\hline & & Macrocentrus sp. & 6 & 0 & 0 & 6 \\
\hline & Formicidae & Camponotus maculatus & 118 & 41 & 16 & 175 \\
\hline
\end{tabular}




\begin{tabular}{|c|c|c|c|c|c|c|}
\hline & & $\begin{array}{c}\text { Camponotus perrisi } \\
\text { Forel. }\end{array}$ & 0 & 1 & 0 & I \\
\hline & & $\begin{array}{c}\text { Camponotus vestitus } \\
\text { Smith }\end{array}$ & 5 & 5 & 2 & 12 \\
\hline & & Camponotus sp. & 65 & 24 & 33 & 122 \\
\hline & & Dorylus sp. & 7 & 4 & 1 & 12 \\
\hline & Ichneumonoidae & Goryphus sp. & 125 & 100 & 122 & 347 \\
\hline Isoptera & Termitidae & Termes sp. & 104 & 36 & 210 & 350 \\
\hline \multirow[t]{6}{*}{ Lepidoptera } & Arctiidae & Eilema sp. & 76 & 63 & 52 & 193 \\
\hline & & Metatarcta sp. & 12 & 12 & 8 & 32 \\
\hline & & Ovenna sp. & 95 & 95 & 108 & 298 \\
\hline & & Spilosoma sp. & 25 & 23 & 15 & 63 \\
\hline & Geometridae & Heterocrita sp. & 50 & 30 & 39 & 119 \\
\hline & Saturniidae & Pseudantheraea sp. & 1 & 0 & 0 & 1 \\
\hline
\end{tabular}

Contine: Table 1

\begin{tabular}{|c|c|c|c|c|c|c|}
\hline Order & Family & Genus/species & RA & OF & AG & Total \\
\hline \multirow[t]{4}{*}{ Mantodea } & Amorphoselidae & Amorphoscelis sp. & 23 & 15 & 16 & 54 \\
\hline & Mantidae & $\begin{array}{c}\text { Empusa sp. } \\
\text { Hoplocorypha }\end{array}$ & 1 & 1 & 1 & 3 \\
\hline & & nigerica Beir. & 3 & 9 & 12 & 24 \\
\hline & & Pygromantis sp. & 0 & 4 & 23 & 27 \\
\hline \multirow[t]{7}{*}{ Orthoptera } & Acrididae & $\begin{array}{c}\text { Eurycorypha sp. } \\
\text { Gastrimargus }\end{array}$ & 0 & 1 & 1 & 2 \\
\hline & & $\begin{array}{c}\text { amplus Sjost. } \\
\text { Oedaleus nigeriensis } \\
\text { Uvarov. }\end{array}$ & 2 & 1 & 2 & 5 \\
\hline & & & 1 & 5 & 1 & 7 \\
\hline & Gryllidae & $\begin{array}{c}\text { Stobbea sp. } \\
\text { Gryllus bimaculatus } \\
\text { De Geer }\end{array}$ & 2 & 0 & 18 & 20 \\
\hline & & & 0 & 0 & 1 & 1 \\
\hline & & $\begin{array}{l}\text { Gymnogryllus sp. } \\
\text { Scapsipedus } \\
\text { marginatus Afz \& }\end{array}$ & 1 & 0 & 1 & 2 \\
\hline & & $\begin{array}{c}\text { Bra } \\
\text { Total }\end{array}$ & $\begin{array}{c}1 \\
\mathbf{1}, 421\end{array}$ & $\begin{array}{c}1 \\
1,283\end{array}$ & $\begin{array}{c}0 \\
1,797\end{array}$ & $\begin{array}{c}2 \\
\mathbf{4 , 5 0 1}\end{array}$ \\
\hline
\end{tabular}

RA - Residential Area

OF - Open Field

AG - Agroecosystem

\section{Relative abundance of insects in the study area}

Table 2. Show the relative abundance of the insect species in the selected habitats Heteronychus mossambicus, had the highest relative abundance of (11.44\%) followed by Termes sp. with (7.78\%) and Goryphus sp (7.71\%). Insect such as Chaenius decipiens, Cheilomenes sulphurea, Copris sp., Cicindela sp. and Derobrachus geminatus have the least abundance of (0.02) each.

Table 3 shows the pooled relative abundance of insects based on orders. The Coleopteran insects have the highest relative abundance (46.42\%) followed by Hymenoptera (18.59\%) and the least is Orthoptera (0.84\%). The diversity indices shows that Coleopteran insects have the highest diversity index $\left(\mathrm{H}^{\prime}=2.547\right)$ and species richness $(\mathrm{d}=2.65)$. Isoptera have 
the least $\left(H^{\prime}=0\right)$ and $(d=0)$. Isoptera was noted to have the highest evenness index $\left(E^{\prime}=1\right)$ and have no equitability.

However, Dictyoptera which have the second highest evenness after Isoptera, have evenness (E') of 0.9484 and the highest equitability of 0.9614 . Diptera have the least evenness and equitability; $\left(E^{\prime}=0.372\right)$ and $(J=0.5706)$.

Table 2. Relative abundance of insect species recovered from selected habitats in Wukari

\begin{tabular}{|c|c|c|}
\hline Order & Genus/Species & Relative Abundance (\%) \\
\hline Dictyoptera & $\begin{array}{c}\text { Anomala mixta } \\
\text { Alcidodes brevirostris } \\
\text { Aulacoryssus aciculatus } \\
\text { Aulacoryssus sp. } \\
\text { Aspidomorpha nigromaculata } \\
\text { Arsinoe biguttata } \\
\text { Chlaenius decipiens } \\
\text { Cheilomenes sulphurea } \\
\text { Callida sp. } \\
\text { Copris sp. } \\
\text { Cicindela sp. } \\
\text { Callida decora } \\
\text { Colliuris sp. } \\
\text { Cylas brunneus } \\
\text { Derobrachus geminatus } \\
\text { Dichaetochilus vagans } \\
\text { Disonycha sp. } \\
\text { Derophaerus sp. } \\
\text { Egadroma sp. } \\
\text { Hister sp. } \\
\text { Heteronychus mossambicus } \\
\text { Melanoxanthus sp. } \\
\text { Onthophagus sp. } \\
\text { Paussus sp. } \\
\text { Prosephus sp. } \\
\text { Paroeme nigripes } \\
\text { Strobiderus sp. } \\
\text { Serica sp. } \\
\text { Schizonycha africana } \\
\text { Tenebriodes sp. } \\
\text { Blattella sp. } \\
\text { Deropeltis sp. } \\
\text { Gyna costalis } \\
\end{array}$ & $\begin{array}{c}3.33 \\
0.28 \\
0.68 \\
1.26 \\
0.11 \\
2.27 \\
0.02 \\
0.02 \\
0.04 \\
0.02 \\
0.02 \\
0.58 \\
1.26 \\
0.78 \\
0.02 \\
1.33 \\
2.17 \\
0.28 \\
0.68 \\
0.53 \\
11.44 \\
0.13 \\
4.42 \\
0.18 \\
2.8 \\
4.09 \\
1.56 \\
2.51 \\
2.02 \\
1.58 \\
0.38 \\
0.44 \\
0.73 \\
\end{array}$ \\
\hline \multicolumn{3}{|c|}{ Continue: Table 2} \\
\hline Order & Genus/Species & Relative Abundance (\%) \\
\hline Diptera & $\begin{array}{c}\text { Acrodesmia pennicornis } \\
\text { Chrysomyia albiceps } \\
\text { Glaurocara townsendi } \\
\text { Latiginella rufogrisea } \\
\text { Musca sorbens } \\
\text { Musca sp. } \\
\text { Musca domestica } \\
\text { Morellia nilotica } \\
\text { Ommatius sp. }\end{array}$ & $\begin{array}{l}0.04 \\
0.15 \\
0.02 \\
1.78 \\
0.13 \\
0.04 \\
1.71 \\
0.04 \\
0.15\end{array}$ \\
\hline
\end{tabular}




\begin{tabular}{|c|c|c|}
\hline & Scarcophaga sp. & 0.07 \\
\hline \multirow[t]{5}{*}{ Hemiptera } & Aspavia acuminata & 0.07 \\
\hline & Coranus lugubris & 0.11 \\
\hline & Cryptoflata unipunctata & 0.13 \\
\hline & Oncocephalus sp. & 0.15 \\
\hline & Piezodorus sp. & 1.22 \\
\hline \multirow[t]{11}{*}{ Hymenoptera } & Apanteles sp. & 0.33 \\
\hline & Braunsia sp. & 0.09 \\
\hline & Camponotus perrisi & 0.02 \\
\hline & Camponotus vestitus & 0.26 \\
\hline & Camponotus maculatus & 3.89 \\
\hline & Camponotus sp. & 2.71 \\
\hline & Dorylus sp. & 0.27 \\
\hline & Goryphus sp. & 7.71 \\
\hline & Halictus sp. & 0.67 \\
\hline & Iphiaulax sp. & 2.51 \\
\hline & Macrocentrus sp. & 0.13 \\
\hline \multirow[t]{6}{*}{ Lepidoptera } & Eilema sp. & 4.29 \\
\hline & Heterocrita sp. & 2.64 \\
\hline & Metatarcta sp. & 0.71 \\
\hline & Ovenna sp. & 6.62 \\
\hline & Pseudantheraea sp. & 0.02 \\
\hline & Spilosoma sp. & 1.40 \\
\hline \multirow[t]{4}{*}{ Mantodea } & Amorphoscelis sp. & 1.20 \\
\hline & Hoplocorypha nigerica & 0.53 \\
\hline & Pygromantis sp. & 0.60 \\
\hline & Empusa sp. & 0.07 \\
\hline \multicolumn{3}{|c|}{ Continue: Table 2} \\
\hline Order & Genus/Species & Relative Abundance (\%) \\
\hline Isoptera & Termes sp. & 7.78 \\
\hline \multirow[t]{7}{*}{ Orthoptera } & Eurycorpha sp. & 0.04 \\
\hline & Gastrimargus amplus & 0.11 \\
\hline & Gryllus bimaculatus & 0.02 \\
\hline & Gymnogryllus sp. & 0.04 \\
\hline & Oedaleus nigeriensis & 0.15 \\
\hline & Scapsipedus marginatus & 0.04 \\
\hline & Stobbea sp. & 0.44 \\
\hline
\end{tabular}

Table 3. Relative abundance of insect orders recovered from selected habitats in Wukari, Taraba State

\begin{tabular}{ccccccc}
\hline Order & $\begin{array}{c}\text { Relative } \\
\text { Abundance } \\
(\%)\end{array}$ & $\begin{array}{c}\text { Community } \\
\text { Dominance } \\
(\%)\end{array}$ & $\begin{array}{c}\text { Shannon } \\
\text { Index (H) }\end{array}$ & $\begin{array}{c}\text { Evenness } \\
\left(\mathbf{E}^{\mathbf{I}}\right)\end{array}$ & $\begin{array}{c}\text { Margalef } \\
(\mathbf{d})\end{array}$ & Equitability (J) \\
\hline $\begin{array}{c}\text { Coleoptera } \\
\text { Dictyoptera }\end{array}$ & 46.41 & 11.65 & 2.547 & 0.6082 & 2.65 & \\
$\begin{array}{c}\text { Diptera } \\
\text { Hemiptera }\end{array}$ & 1.55 & 36.29 & 1.056 & 0.9484 & 0.4708 & 0.8367 \\
Hymenoptera & 1.68 & 36.05 & 1.314 & 0.372 & 1.722 & 0.5714 \\
Isoptera & 18.59 & 50.52 & 1.086 & 0.4936 & 1.144 & 0.606 \\
Lepidoptera & 1.78 & 15.72 & 1.625 & 0.4618 & 1.486 & 0.6778 \\
Mantodea & 15.68 & 29.13 & 1.384 & 0.6651 & 0.7622 & 0.7724 \\
Orthoptera & 2.4 & 36.27 & 1.127 & 0.7715 & 0.6407 & 0.8129 \\
\hline
\end{tabular}




\section{Diversity indices of insects in the study area}

Table 4a shows that agro ecosystem have the highest relative abundance of insects (39.91\%), while the open field have the least (28.51\%). The Fisher - alpha diversity indices shows that the farm has the highest index of diversity (14.24) while, the residential has the least (11.9)

The open field have the least species richness $(\mathrm{d}=7.685)$ but, has the highest diversity $\left(\mathrm{H}^{\prime}=\right.$ $3.345)$, evenness $\left(E^{\prime}=0.5617\right)$ and, equitability $(\mathrm{J}=0.8565)$. However, the agro ecosystem have the highest species richness $(\mathrm{d}=9.074)$.

The t- test analysis on the Shannon diversity index shows there is a significant difference $(\mathrm{P}<$ 0.05) between RA and OF, RA and AG in terms of species diversity. However, there is no significant difference $(\mathrm{P}>0.05$ ) between $\mathrm{OF}$ and $\mathrm{AG}$ in term of species diversity (Table 5 ).

Table 6 shows the Jaccard similarity index values. The levels of species similarity between the habitats surveyed are high as they are all above 0.5 . However, the highest similarity index was observed between residential area and the agroecosystem (0.718).

Table 4 a. Diversity indices on insect species recovered from the selected habitats in Wukari

\begin{tabular}{ccc}
\hline Location & Relative Abundance (\%) & Fisher - alpha $(\boldsymbol{\alpha})$ \\
\hline Residential Area & 31.57 & 11.9 \\
Open Field & 28.51 & 11.95 \\
Agro ecosystem & 39.91 & 14.24 \\
\hline
\end{tabular}

Table 4 b. Diversity indices on insect species recovered from the selected habitats in Wukari

\begin{tabular}{cccccc}
\hline Location & $\begin{array}{c}\text { Community } \\
\text { Dominance } \\
(\%)\end{array}$ & $\begin{array}{c}\text { Shannon Index } \\
\left(\mathbf{H}^{\prime}\right)\end{array}$ & Evenness (E') & Margalef (d) & Equitability (J) \\
\hline Residential Area & 4.96 & 3.316 & 0.4835 & 7.714 & \\
Open Field & 4.23 & 3.345 & 0.5617 & 7.685 & 0.8202 \\
Agro ecosystem & 5.78 & 3.344 & 0.4107 & 9.074 & 0.7868 \\
\hline
\end{tabular}

Table 5. P value for T-test showing the level of difference in insect diversity between the study habitats

\begin{tabular}{lccc}
\hline & RA & AG & OF \\
\hline RA & $0.005^{*}$ & $0.0002^{*}$ \\
AG & & $0.45 \mathrm{NS}$ \\
OF & & \\
\hline
\end{tabular}

RA -Residential Are, AG- Agro ecosystem, OF - Open Field

* - Significantly different at $5 \%$ level of significance

NS - Not Significantly different at 5\% level of significance

Table 6. Jaccard similarity index values

\begin{tabular}{lccc}
\hline & RA & AG & OF \\
\hline RA & 1 & 0.718 & 0.6911 \\
AG & & 1 & 0.689 \\
OF & & & 1 \\
\hline
\end{tabular}

RA -Residential Area, A G- Agro ecosystem, OF- Open Field

* - Significantly different at $5 \%$ level of significance 


\section{Economic importance of insects in the study area}

Table 7 shows that 2 of the overall dominant insect species are beneficial serving as natural enemies of insect pests and soil formation and aeration and protein source for man. Table 8 shows that the rare species are made up of beneficial and pestiferous insect species.

Table 7. Economic importance of dominant insect species in the study area

\begin{tabular}{cc}
\hline Insect Species & Economic importance \\
\hline Heteronchyus mossambicus & Pest of crops \\
Goryphus sp. & Parasitoid \\
Termes sp. & Entomophagy/Soil formation \\
\hline
\end{tabular}

Table 8. Economic importance of rare insect species in the study area

\begin{tabular}{cc}
\hline Insect Species & Economic importance \\
\hline Cicindela sp. & Predator \\
Chlaenius decipiens & Predator \\
Camponotus perrisi & Predator \\
Cheilomenes sulphurea & Predator \\
Copris sp. & Decomposer \\
Derobranchus geminatus & Pest of crops \\
Gryllus bimaculatus & Pest crops \\
Pseudantheraea sp. & Pest of crops \\
Glaurocara townsendi & Parasitoid \\
\hline
\end{tabular}

\section{Discussion}

\section{Diversity and abundance of insects in the study area}

A total of 9 orders, 34 families and 77 insect species were found in the habitats surveyed in Wukari. A total of 4,501 individual insect species was collected during the survey period using pitfall, light and yellow pan traps. Different trapping methods were used to attract different kinds of insects. This is in line with the report of John, 1989 who reported that using a combination of traps gives better species richness data.

The most abundant insect overall was Heteronychus mossambicus followed by Termes sp. and Goryphus sp. Insects species such as Chlaenius decipiens, Cheilomenes sulphurea, Copris sp., Cicindela sp. Pseudantheraea sp., Derobranchus geminatus, Glaurocara townsendi, Camponotus perrisi, and Gryllus bimaculatus were rarely found.

\section{Relative abundance of insect orders in the study area}

Overall, Coleoptera was the most abundant (46.41\%) insect order in the study area. This was followed by Hymenoptera (18.59\%), Lepidoptera (15.68\%) and the least; Orthoptera $(0.84 \%)$. This agrees with the report of Tscharntke and Brandl, 2004 who acknowledges coleopterans as the most predominant insect order.

\section{Diversity indices of insect orders}

Diversity indices shows that Coleoptera is the most diverse (Shannon $\mathrm{H}^{\prime}=2.547$ ) and has a high evenness and equitability indices (0.6082 and 0.8367) with the report of (Bradshaw et al., 2009) on high diversity of coleopterans in tropical environments. 


\section{Diversity indices on selected habitats}

Overall, the agro ecosystem was notably the highest in terms of species diversity ( $\alpha=$ $14.24)$ and richness $(d=9.074)$. The least is the residential area; $(\alpha=11.9)$ and $(d=7.714)$. Therefore as plant species increases, insect species also increase. This agrees with the reports (Gaston, 1991; David et al., 1994; Cheng et al., 2007) that substantiated that plants and insects interact by way of mutualism and phytophagy. The highest similarity was observed between the agro ecosystem and residential area with $71.8 \%$ overlap. However, the t'-test statistical analysis shows no significant difference in species diversity between the open field (grassland) and the agro ecosystem. This can be understood from the standpoint that, both communities are highly plant based and plants have been believed to co-evolve with their insect herbivores (David et al., 1994; Tscharntke et al., 2004). They are also found where there is a favorable condition for their survival (Samways, 2007; Adetundan et al., 2013).

\section{Economic importance of insects in the study area}

Each insect plays an ecosystem service and contributes to the stability of the ecosystem. The dominant and rare species were noted to cut across beneficial and noxious species. This is in agreement with (Maina et al., 2012).

\section{Conclusion}

The survey has shown that Wukari is rich in insect biodiversity. It has also documented probably for the very first time, the insect fauna in wukari. This information will assist all stakeholders to optimize the beneficial amidst them while, managing noxious species. Further studies should be conducted using other sampling techniques and by also expanding the geographical scope of the study. There is need to also expand the duration of the study as seasonal variations affect population dynamics of insects.

\section{Acknowledgements}

The researchers appreciate the support of Mr. Ishaku Musa, of the Institute of Agriculatural Research (IAR), Ahmadu Bello University, Zaria, Kaduna State, Nigeria for patiently taking his time to identify the insect samples.

\section{References}

Adetundan, SA, Olusola, JA. (2013). Diversity and abundance of arthropod and tree species as influenced by different forest vegetation types in Ondo State Nigeria, Int J Ecosystem, 3(3):19-23.

Adetundan, SA, Ofuya, TI, Fuwape, JA. (2005). Environmental effects of insects herbivores and logging on tree species diversity in Akure Forest Reserve (Apomu)", Nigeria Appl Trop Agric, 9(1\&2):12-18.

Axmacher, JC, Fiedler, K. (2004). Manual versus automatic moth sampling at equal light sources: A comparison of catches from mountain Kilimanjaro, J Lepidopt Soc, 58:196-202. 
Berenhaum, MR. (1995). Bugs in the System: Insect and their Impact on Human Affair, Addison - Wesley publishing, p. 296.

Bradshaw, CJA, Sohdi NS, Brook, BW. (2009). Tropical turmoil a biodiversity tragedy in progress, Front Ecol Environ, 7:79- 87.

Campos, WG, Pereira DBS, Schoerder, JH. (2000). Comparison of the efficiency of flight interception trap models for sampling hymenoptera and other insects", Anais da Soc Entomol do Bra, 29:381- 389.

Cheng, S, Kirton, LG. (2005). Overview of insect biodiversity research in peninsular Malaysia, pp 121- 128. In Chua, L.SL, L.G Kirton and L.G, Saw (eds). Status of biodiversity in Malaysia and threat assessments of plant species in Malaysia. Proceeding of the seminar and workshop, 28-30.

David, HE, Mark, DR. (1994). Interactions between herbivores insects and plant insect coevolution, America Natur, 145(4):677-697.

Frank, KD. (2006). Effect of Artificial Light on Moth. In: Ecological Consequence of Artificial Night Lighting (Longcore T, Rich C, Eds.), Washington DC: Island press, pp. 305-344.

Gaston, KJ. (1991). The magnitude of global insect species richness", Conservat Biology, 5:283-296.

Godfray, HC. (2002). Challenges for taxonomy, Nature, 417:17-19.

John, SN. (1989). A study of five methods of sampling Hymenoptera (Insecta) in a tropical rainforest, with reference to the Parasita, J Natur History, 23:285-298.

Kato, M, Itioka, T, Sakai, S, Monose, K, Yamane, S. Hamid AA, Inoue, T. (2000). Various population fluctuation patterns of light attracted beetle in a tropical lowland dipterocarp forest in Sarawak, Populat Ecology, 42:97-104.

Maina, YT, Maina, UM. (2012). Preliminary survey of insect fauna around the Lake Chad basin area of Borno State, Nigeria, J Natur Sci Res, 2(3):75-79.

Mazon M, Bordera, S. (2008). Effectiveness of two sampling methods used for collecting Tchneumonidae (hymenoptera) in the cabaneros national park (spain), Eur J Entomol, 105:879-888.

Meddler, JT. (1980). Insect of Nigeria checklist and bibiolography", Memoir of American Entomological Institute, p. 919.

Miller SE, Rogo, LM. (2001). Challenges and oppuntunities in understanding and utilization of African insect diversity, Cimbebasia, 17:197-218.

Premalatha, M, Abbasi, T, Abbasi, T, Abassi, SA. (2011). Energy efficient food production to reduce global warming ecodegradation: The use of edible insects, Renew Sustain Energy Rev, 15(9):4357-4360. 
Roulston, TH, Smith, SA, Brewster, AL. (2007). A comparison of pan trap and intensive net sampling techniques for documenting bees (Hymenoptera: Apiformes) fauna, J Kansas Entomol Soc, 80:179-181.

Sabu, TK, Shiju, RT. (2010). Efficiency of pitfall trapping; winker and berlese extraction methods for measuring ground-dwelling arthropods in moist deciduous forests in Western Ghats, J Insect Sci, vol. 10, pp. 1-17.

Samways, MJ. (2007). Connecting biodiversity, Trend in Ecology and Evolution, 22:60.

Saunder, ME, Luck, GW. (2013). Pan trap catches of pollinators insects vary with habit". Australia J Entomol, 52:106-113.

Tscharntke, T, Brandl, R. (2004). Plant insect interactions in fragmented landscapes", Ann Rev Entomol, 49:405-430.

Wardle, DA. (2002). Community and Ecosystem linking the above and below ground components. Princeton university press, Princeton New Jersey, p. 392.

Winder, L, Holland, JM, Perry, J, Woolley NC, Alexander, CJ. (2001). The use of barrier connected pitfall trapping for sampling predatory beetles and spider", Entomol Experimen Appl, 98:249-258.

Yi, Z, Jinchao, F, Dayuan, X, Weiguo S, Axmacher, JC. (2012). A Comparison of terrestrial arthropods sampling methods, J Res Ecology, 3:174-182.

How to cite this article: Okrikata Emmanuel, Yusuf Olajumoke Anuoluwa, A Study on the Diversity and Relative Abundance of Insect Fauna in Wukari, Taraba State, Nigeria. International Journal of Advanced Biological and Biomedical Research, 2019, 7(2), 129141. Link: http://www.ijabbr.com/article 34369.html 